\title{
Optimal transformation of frequency response functions on interface deformation modes
}

\author{
M. Häußler ${ }^{\mathrm{a}}$ and D. J. Rixen ${ }^{\mathrm{b}}$ \\ ${ }^{\mathrm{a}, \mathrm{b}}$ Institute of Applied Mechanics, Faculty of Mechanical Engineering, Technische Universität München, \\ Boltzmannstr. 15, 85748 Garching, Germany \\ amichael.haeussler@tum.de, brixen@tum.de
}

\begin{abstract}
Frequency based dynamic substructuring (FBS) allows to predict the dynamic behavior of a complex system where neither building a physical prototype of the assembled system, nor possessing a detailed numerical model of all substructures is required. A task that frequently arises in engineering practice when developing a product containing many supplier parts. However, in the experimental realm, modeling the interface connection between two substructures is not as straightforward as in numerical analysis. The consideration of rotational degrees of freedom (rdof) on the interface seems to be crucial for accurate results, but no common procedure has been established yet. By projecting measured sensor data on interface deformation modes (IDMs) it is possible to consider rdof as well as filtering out uncorrelated measurement noise. The transformation of a measured frequency response function (FRF) matrix on some generalized IDMs has recently been derived by directly using Moore-Penrose pseudoinverses. The transformation process can also be seen as a minimization procedure, e.g. as simple least squares for the displacements and a convex optimization for the forces.

This contribution derives the pseudoinverses starting from this minimization point of view, where the engineer is free to choose the quantity to be minimized. From this interpretation, some suggestions for including more engineering judgment in the transformation are made (either gained during testing practice, from measurement variances or mechanical energy minimization principles). We also show that the coupling of transformed FRF matrices effectively corresponds to a weakening of the interface compatibility conditions.

Thereby, we hope to give some insight in the meaning of the weighting matrices involved in the transformation, and provide a framework for deriving improved coupling methods in the future.
\end{abstract}

Keywods: experimental dynamic substructuring, virtual point transformation, optimal projection, interface weakening.

\section{Nomenclature:}

$\begin{array}{llll}\mathbf{u}, \mathbf{f} & \text { measured displacements / forces } & \mathbf{T} & \text { transformation matrix } \\ \mathbf{q}, \mathbf{m} & \text { generalized displacements / forces } & \mathbf{\Sigma} & \text { covariance matrix } \\ \mathbf{Y}, \mathbf{Z} & \text { admittance / impedance matrix } & { }_{I} \mathbf{e} & \text { unit vector measured in coord. system } I \\ \mathbf{B} & \text { signed Boolean matrix } & \tilde{\mathbf{f}}_{2} & \text { equivalent interface forces } \\ \boldsymbol{\lambda} & \text { Lagrange multiplier vector } & (\star)^{A} & \text { quantity pertaining to substructure A } \\ \mathbf{R} & \text { interface deformation mode matrix } & (\star)^{A \mid B} & \text { uncoupled block notation of } A \text { and } B \\ \mathbf{W} & \text { weighting matrix } & (\star)^{A B} & \text { coupled quantity of } A \text { and } B \\ \boldsymbol{\mu} & \text { residual in displacement transformation } & (\star)_{1} & \text { pertaining to set of dofs } 1 \\ \Phi(\star) & \text { cost function } & (\star)_{u / f} & \text { pertaining to disp./force transformation } \\ & & & \\ \text { DS } & \text { dynamic substructuring } & \text { FBS } & \text { frequency based substructuring } \\ \text { FRF } & \text { frequency response function } & \text { IDM } & \text { interface deformation mode } \\ \text { VPT } & \text { virtual point transformation } & \text { SEREP } & \text { system equvalent reduction and expansion } \\ \text { EMPC } & \text { equivalent multiple point connection } & \text { dof } & \text { degree of freedom } \\ \text { CMS } & \text { component mode synthesis } & \text { rdof } & \text { rotational degree of freedom }\end{array}$



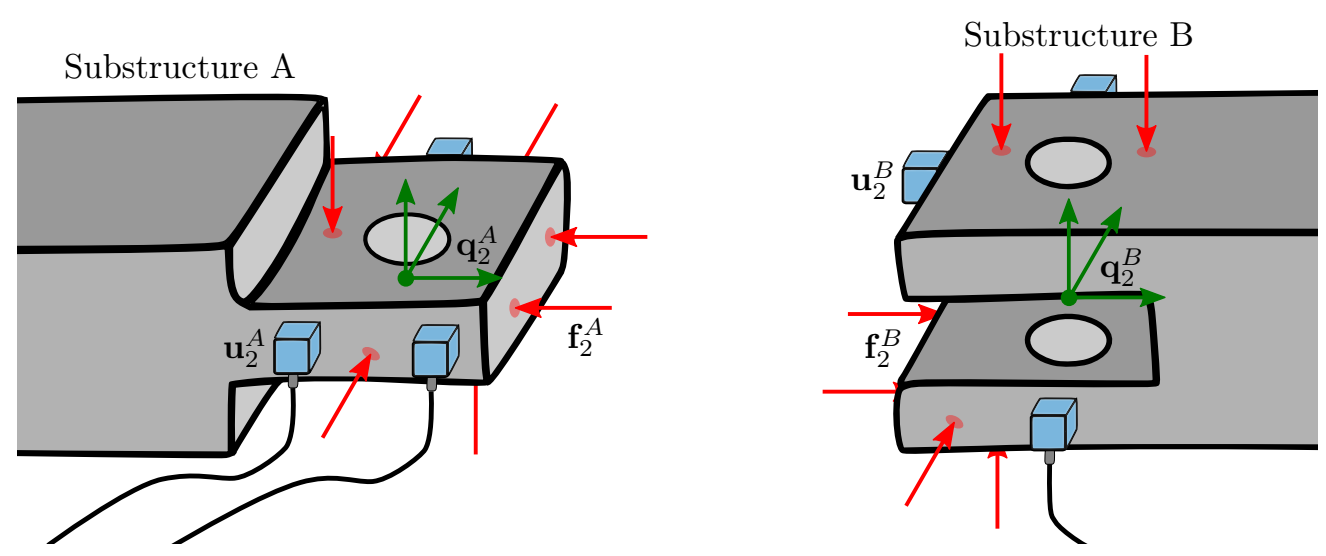

Figure 1: Assembly problem in experimental dynamic substructuring. Sensors $\mathbf{u}_{2}$ and force inputs $\mathbf{f}_{2}$ on either side of the interface are non-matching.

\section{Introduction}

Dynamic Substructuring (DS) has become a well accepted method that provides a framework for numerically assembling subcomponents, developed by different design teams, and predict the dynamic behavior of the complete product. Variants for coupling in different domains (e.g. the physical-, modal-, frequency -domain, etc.) can be derived straight forward [1] and can equivalently be used for decoupling of structures [2,3]. The frequency based substructuring (FBS) method was first published by Jetmundsen [4] and is well suited for experimental dynamics (although similar methods where proposed earlier [5]). However, the definition of the interface coupling in experimental DS remains challenging. On the one hand the measured degrees of freedom (dofs) on either side of the interface are often non-matching due to practical instrumentation problems (see substructure A and B in figure 1). They can thus not be coupled by simply enforcing equality in each dof, as in numerical analysis.

Another problem involves measuring and exciting rotational degrees of freedom (rdof) on the interface. Sensors that can actually measure rdof have been discussed for a long time and can even be found in textbooks [6, p.166ff] and [7, p.197ff]. However, rotational acceleration sensors are still exotic and not commonly available. Nevertheless, including rdof in the coupling process has been shown to be crucial for accurate results $[8,9]$.

Therefore, different methods have been developed to implicitly measure and include rotations in the coupling. A large class of methods tries to infer the rotational motion on the interface by using global vibration modes of the substructures to be coupled. They are thus closely related to the component mode synthesis (CMS) techniques, well known from numerical modeling [10]. The 'system equivalent reduction and expansion' (SEREP) procedure [11] is an elegant way to infer full motion data of the structure (including rdof) by measuring with translational sensors only at a few measurement points. An application to experimental DS can be found in [12]. An advantage of these techniques is that the mode identification processes has a 'smoothing effect' on the frequency response functions that are being coupled, thus avoiding many of the numerical instability issues that are common in FBS [13]. However, these methods have to find the global modes of the structure either by building a finite element model or performing an experimental modal analysis. In order to account for the effect of higher order modes in the frequency range of interest, one is usually extending the frequency range to include some of those higher order modes in the model and add a residual flexibility term for even higher modes [9].

For complex systems (e.g. mechatronic components in a car), the modal density might be too high for effectively performing substructuring methods based on global vibration modes and a frequency based approach is preferable. One advantage of directly using frequency response functions (FRFs) is that the measurement data already contains residual terms from higher order modes. To account for rdof implicitly some methods have used multiple connection points on the interface that are simultaneously coupled [14, 9], which has been called 'equivalent multiple point connection' (EMPC) [8]. However, coupling too many points can result in an overdetermination of the interface coupling equations, making some of the equations almost redundant and cause numerical instabilities. Additionally the direct use of experimentally obtained data in the FRFs inherently causes numerical instabilities [13]. An interesting depiction of different methods, their problems and remedies can be found in the companion publications $[2,14]$, where it was found that enforcing the coupling in only a 'weak' least squares sense in many (modal) dofs, can significantly improve the substructuring results.

A recent approach that can be seen as a combination of the CMS and EMPC methods above, is using 'interface deformation modes' (IDMs) which aren't global vibration modes but rather kinematic assumptions of the local deformation behavior directly at the interface. The IDMs can be constructed without the need of building a finite 


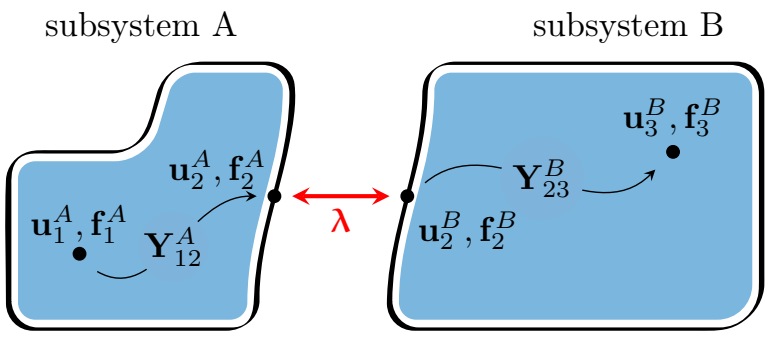

(a) Admittances of uncoupled subsystems A and B.

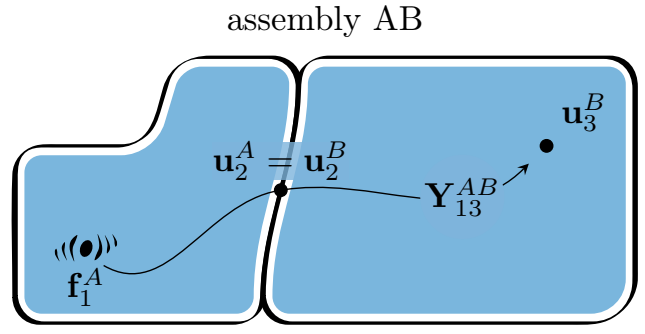

(b) Admittance of coupled assembly AB.

Figure 2: The substructuring problem [17].

element model (which might be impossible due to the lack of information for supplier parts) or performing an experimental modal analysis (which might be infeasible due to a high modal density). However, the transformation of measured FRFs on IDMs can also provide some numerical smoothing if the transformation is overdetermined. This method has first been used in [8] with driving point FRFs and was extended in [15] to allow for different positions in sensors and force impacts, which is making it more feasible experimentally (see figure 1). In [16] the transformation matrices necessary for transforming the measured FRF matrices on generalized IDMs where derived using Moore-Penrose pseudoinverses, that allow for a different weighting of physical dofs in the transformation on the IDMs. In this contribution we will derive the transformation matrices anew from a minimization point of view and show how the weighting matrices can be utilized to further improve the transformation and coupling process. We will additionally show that the transformation of the physical dofs also has a 'weakening' effect and is enforcing the compatibility only in a 'least squares' sense.

\section{Theory}

This section gives a short review on how to couple the admittances of two substructures with matching dofs. Subsequently the transformation of measured sensor signals $\mathbf{u}_{2}$ on generalized IDMs $\mathbf{q}$, and the expansion of generalized forces $\mathbf{m}$ on the measured force impacts $\mathbf{f}_{2}$ is derived from the minimization of a cost function. This cost function can be chosen to include some optimality criterion. Concluding, the effect of coupling the transformed $\mathbf{q}, \mathbf{m}$ of both substructures, instead of coupling the measured quantities $\mathbf{u}_{2}, \mathbf{f}_{2}$ directly, is shown to act as a weakening of the interface compatibility conditions.

\subsection{Frequency Based Substructuring}

Frequency based substructuring (FBS) aims at deriving the admittance of an assembled system $\mathbf{Y}^{A B}$ from the separate admittances of two subsystems $\mathbf{Y}^{A}$ and $\mathbf{Y}^{B}$. Consider the situation depicted in figure 2. The admittances of both subsystems $\mathbf{Y}^{A}$ and $\mathbf{Y}^{B}$ are known, where their dofs are grouped into some internal dofs $\left((\star)_{1}^{A}\right.$ and $\left.(\star)_{3}^{B}\right)$ and some common dofs on the interface $\left((\star)_{2}^{A}\right.$ and $\left.(\star)_{2}^{B}\right)$. Displacements are denoted by $\mathbf{u}$, external forces in the respective dofs are denoted by $\mathbf{f}$. The admittance of the uncoupled substructures can be written in block diagonal form $\mathbf{Y}^{A \mid B}$. The following equation is the starting point for coupling $\mathbf{Y}^{A}$ and $\mathbf{Y}^{B}$, but also aims at clarifying the notation in verbose and compact form:

$$
\mathbf{Y}^{A \mid B}(\mathbf{f}+\mathbf{g})=\left[\begin{array}{cc}
\mathbf{Y}^{A} & \mathbf{0} \\
\mathbf{0} & \mathbf{Y}^{B}
\end{array}\right]\left(\left[\begin{array}{l}
\mathbf{f}^{A} \\
\mathbf{f}^{B}
\end{array}\right]+\left[\begin{array}{l}
\mathbf{g}^{A} \\
\mathbf{g}^{B}
\end{array}\right]\right)=\left[\begin{array}{cccc}
\mathbf{Y}_{11}^{A} & \mathbf{Y}_{12}^{A} & \mathbf{0} & \mathbf{0} \\
\mathbf{Y}_{21}^{A} & \mathbf{Y}_{22}^{A} & \mathbf{0} & \mathbf{0} \\
\mathbf{0} & \mathbf{0} & \mathbf{Y}_{22}^{B} & \mathbf{Y}_{23}^{A} \\
\mathbf{0} & \mathbf{0} & \mathbf{Y}_{32}^{B} & \mathbf{Y}_{33}^{A}
\end{array}\right]\left(\left[\begin{array}{c}
\mathbf{f}_{1}^{A} \\
\mathbf{f}_{2}^{A} \\
\mathbf{f}_{2}^{B} \\
\mathbf{f}_{3}^{B}
\end{array}\right]+\left[\begin{array}{c}
\mathbf{0} \\
\mathbf{g}_{2}^{A} \\
\mathbf{g}_{2}^{B} \\
\mathbf{0}
\end{array}\right]\right)=\left[\begin{array}{c}
\mathbf{u}_{1}^{A} \\
\mathbf{u}_{2}^{A} \\
\mathbf{u}_{2}^{B} \\
\mathbf{u}_{3}^{B}
\end{array}\right]=\mathbf{u}
$$

where $\mathbf{g}$ denotes the yet unknown reaction forces acting between the two substructures in the coupled state. When coupled, the two substrucutures have to fulfill continuity on the interface $\left(\mathbf{u}_{2}^{A}-\mathbf{u}_{2}^{B}=\mathbf{0}\right)$. The reaction forces $\mathbf{g}$ needed for achieving continuity fulfill actio est reactio, i.e. they are equal in magnitude but have opposite signs $\left(\mathbf{g}_{2}^{A}=-\mathbf{g}_{2}^{B}\right)$. They can thus be denoted by a single vector of reaction forces $\boldsymbol{\lambda}$. These conditions result in the following set of equations:

$$
\begin{array}{r}
\mathbf{Y}^{A \mid B}(\mathbf{f}+\underbrace{\mathbf{B}^{T} \boldsymbol{\lambda}}_{=\mathbf{g}})=\mathbf{u} \\
\mathbf{B u}=\mathbf{0}
\end{array}
$$$$
\text { where } \mathbf{B}=\left[\begin{array}{llll}
\mathbf{0} & \mathbf{I} & -\mathbf{I} & \mathbf{0}
\end{array}\right] \text {, }
$$ 
so that the reaction forces in eq. (2) automatically fulfill actio est reactio and the statement in eq. (3) enforces continuity. The matrix B is commonly called a 'signed Boolean matrix'. Inserting eq. (2) in eq. (3) and solving for $\lambda$ :

$$
\boldsymbol{\lambda}=-\underbrace{\left(\mathbf{B} \mathbf{Y}^{A \mid B} \mathbf{B}^{T}\right)^{-1}}_{Z_{i n t}} \underbrace{\mathbf{B Y} \mathbf{Y}^{A \mid B} \mathbf{f}}_{\Delta \mathbf{u}_{2}}
$$

where the term $\Delta \mathbf{u}_{2}$ denotes the interface gap which would result between both structures if they where uncoupled (due to the externally applied forces $\mathbf{f}$ ). The term $\mathbf{Z}_{\text {int }}$ can be interpreted as the 'interface stiffness' that relates the interface gap $\Delta \mathbf{u}_{2}$ to the reaction forces $\boldsymbol{\lambda}$. Reinserting eq. (4) in eq. (2) yields the admittance matrix of the assembled system $\mathbf{Y}^{A B}$ :

$$
\underbrace{\left(\mathbf{Y}^{A \mid B}-\mathbf{Y}^{A \mid B} \mathbf{B}^{T}\left(\mathbf{B} \mathbf{Y}^{A \mid B} \mathbf{B}^{T}\right)^{-1} \mathbf{B} \mathbf{Y}^{A \mid B}\right)}_{=\mathbf{Y}^{A B}} \mathbf{f}=\mathbf{u}
$$

Remark 1: Note that the assembled matrix $\mathbf{Y}^{A B}$ in eq. (5) still has the same size as the unassembled block matrix $\mathbf{Y}^{A \mid B}$. When writing down eq. (5) in the verbose form indicated in eq. (1), we would notice that indeed the second and third row of $\mathbf{Y}^{A B}$ are equal, which is a direct consequence of the continuity $\left(\mathbf{u}_{2}^{A}=\mathbf{u}_{2}^{B}\right)$. Additionally we would notice that the second and third column in $\mathbf{Y}^{A B}$ are equal, meaning that the external forces on the interface $\mathbf{f}_{2}^{A}$ and $\mathbf{f}_{2}^{B}$ both have the same effect on the response of the assembled system. It is thus common practice to remove these redundant rows and columns from the final matrix $\mathbf{Y}^{A B}$, thereby (possibly) making it invertible again and treating the interface dofs as the common quantities they are:

$\mathbf{u}_{2}^{A}=\mathbf{u}_{2}^{B}=: \mathbf{u}_{2}^{A B} \quad$ and $\quad \mathbf{f}_{2}^{A}+\mathbf{f}_{2}^{B}=: \mathbf{f}_{2}^{A B}$.

\subsection{Transformation of FRF Matrices}

Note that we have implicitly assumed that the dofs/sensors on either side of the interface $\mathbf{u}_{2}^{A}$ and $\mathbf{u}_{2}^{B}$ are in the same geometric position and direction once the substructures are coupled. We additionally assumed that the forces $\mathbf{f}_{2}^{A}$ and $\mathbf{f}_{2}^{B}$ attack at the same positions and in the same directions, so that actio est reactio for the reaction forces is justified. As opposed to e.g. finite element modeling, in experimental practice this cannot always be assured (see e.g. figure 1). It is thus desirable to project the measured sensor displacements ${ }^{1} \mathbf{u}_{2}$ and the force inputs $\mathbf{f}_{2}$ on some generalized IDMs. The resulting generalized displacements $\mathbf{q}$ and generalized forces $\mathbf{m}$ are matching on either side of the interface and can thus be coupled as explained in section 2.1. The transformation, derived in this subsection will take the following form [16]:

$$
\mathbf{Y}_{q m}=\mathbf{T}_{u} \mathbf{Y}_{22} \mathbf{T}_{f}^{T}
$$

where $\mathbf{Y}_{22}$ denotes the measured FRF matrix in the nonmatching interface dofs $\mathbf{u}_{2}, \mathbf{f}_{2}$ and $\mathbf{Y}_{q m}$ denotes the transformed FRF matrix in the (matching) generalized coordinates. $\mathbf{T}_{u}$ and $\mathbf{T}_{f}^{T}$ are the transformation matrices:

$$
\mathbf{q}=\mathbf{T}_{u} \mathbf{u}_{2} ; \quad \mathbf{f}_{2}=\mathbf{T}_{f}^{T} \mathbf{m} .
$$

The transformation in this section will only concern the interface submatrices $\mathbf{Y}_{22}$, the transformation of the whole matrix $\mathbf{Y}^{A}$ or $\mathbf{Y}^{B}$ is straight forward (see sec. 2.3). Note that the generalized IDMs can be chosen such, that also substructure models from different domains can be coupled (e.g. a finite element model coupled with an experimentally obtained model).

\subsubsection{Displacement modes}

In the case of nonmatching sensor dofs on the interface $\mathbf{u}_{2}$, we can choose to represent the interface motion by some general interface deformation modes (IDMs) and the corresponding generalized coordinates q. The number of IDMs is typically lower than the number of measured interface dofs:

$$
\mathbf{u}_{\mathbf{2}} \in \mathbb{C}^{n_{u}}, \quad \mathbf{q} \in \mathbb{C}^{p}, \quad \text { with } \quad p \leq n_{u} .
$$

Let us consider the single interface connection point depicted in figure 3. When we assume for example that the interface behaves almost rigid in the frequency range of interest we can compose the generalized coordinates of

\footnotetext{
${ }^{1}$ Sensors are typically measuring acceleration, but for simplicity we are talking of displacements here.
} 


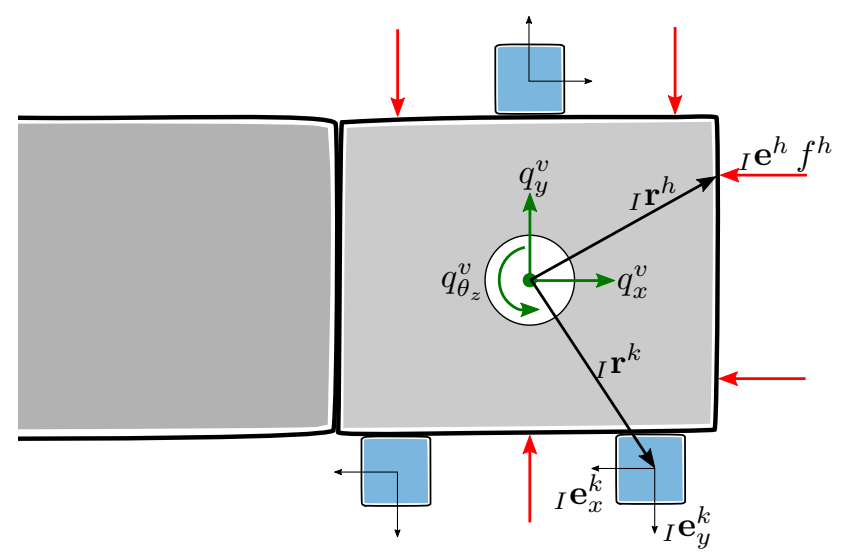

Figure 3: General interface connection point. Exemplary quantities for one sensor $k$ and one force input $h$.

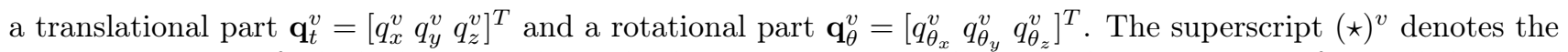
coupling point $v$, of which there may be multiple on a structure. These coupling points are often called virtual points since they are at a geometric position where no physical sensor or input has to be $[15,16,8]$. Let us assume we want to find the displacement $u_{x}^{k}$ measured in $x$-direction of sensor $k$ (see figure 3 ). The relation between the motion in this single sensor channel and the generalized coordinates can easily be found, provided we know the orientation of the sensor $x$-axis ${ }_{I} \mathbf{e}_{x}^{k}$ and the sensors distance from the virtual point ${ }_{I} \mathbf{r}^{k}$, all measured in the global coordinate system $I$ (as indicated by subscript ${ }_{I}(\star)$ ).

$$
u_{x}^{k}=\left({ }_{I} \mathbf{e}_{x}^{k}\right)^{T}\left(\mathbf{q}_{t}^{v}+\mathbf{q}_{\theta}^{v} \times{ }_{I} \mathbf{r}^{k}\right)=\left({ }_{I} \mathbf{e}_{x}^{k}\right)^{T}\left[\begin{array}{cccccc}
1 & 0 & 0 & 0 & { }_{I} r_{z}^{k} & -{ }_{I} r_{y}^{k} \\
0 & 1 & 0 & -{ }_{I} r_{z}^{k} & 0 & { }_{I} r_{x}^{k} \\
0 & 0 & 1 & { }_{I} r_{y}^{k} & -{ }_{I} r_{x}^{k} & 0
\end{array}\right]\left[\begin{array}{c}
q_{x}^{v} \\
q_{y}^{v} \\
q_{z}^{v} \\
q_{\theta_{x}}^{v} \\
q_{\theta_{y}}^{v} \\
q_{\theta_{z}}^{v}
\end{array}\right]=\mathbf{R}_{u_{x}^{k}} \mathbf{q}^{v},
$$

where $\mathbf{R}_{u_{x}^{k}}$ is a $1 \times 6$ matrix relating the generalized coordinates $\mathbf{q}^{v}$ to the displacement in channel $u_{x}^{k}$. The displacements in all sensor channels can be reconstructed for a given $\mathbf{q}^{v}$ following a similar procedure. By stacking all sensor transformation matrices for all virtual points (see [16]), we finally get:

$$
\mathbf{u}_{2}=\mathbf{R}_{u} \mathbf{q} \quad \text { with } \quad \mathbf{R}_{u} \in \mathbb{R}^{n_{u} \times p} .
$$

This can be seen as representing $\mathbf{u}_{2}$ by a reduced basis contained in $\mathbf{R}_{u}$. Note that the reduced basis $\mathbf{R}_{u}$ may contain arbitrary other interface deformation modes, not just the rigid modes for a virtual point as discussed above. One may also want to introduce some interface modes that bend the coupling area or any other modes that appear to be of special importance, e.g. after studying the operational deformation shapes of a structure. One could also approximate the motion of a continuous interface by evaluating some continuous vibration modes at the actual sensor positions and include these modes as a column in $\mathbf{R}_{u}$. However, the general form of (9), i.e. the reduction of sensor dofs will remain the same.

\subsubsection{Displacement transformation}

In reality, the response in the sensor channels $\mathbf{u}_{2}$ will not behave perfectly as indicated in eq. (9), meaning we can only represent a measured $\mathbf{u}_{2}$ with the IDMs up to a certain residual $\boldsymbol{\mu}$.

$$
\mathbf{u}_{2}=\mathbf{R}_{u} \mathbf{q}+\boldsymbol{\mu} \quad \rightarrow \quad \boldsymbol{\mu}=\mathbf{u}_{2}-\mathbf{R}_{u} \mathbf{q}
$$

However, if our kinematic assumption of the interface motion, contained in $\mathbf{R}_{u}$, is good enough in the frequency range of interest (e.g. the rigid body assumption on a virtual point as shown above), then the residual $\boldsymbol{\mu}$ is likely to be very small and containing mostly measurement noise or other uncertainties in experimental practice. See e.g. [16] for some indicator functions that check the validity of the kinematic assumption.

Finding the $\mathbf{q}$ that best approximates the measured response $\mathbf{u}_{2}$ is typically a task for least squares. However we can choose the more general approach to minimize a cost function $\Phi(\mathbf{q})$, which is merely the error vector $\mu$ 
weighted by a weighting matrix $\mathbf{W}_{u}{ }^{2}$. The weighting is chosen from an engineering point of view and some possible choices for $\mathbf{W}_{u}$ are discussed later. The function we want to minimize with the transformation, then is:

$$
\begin{aligned}
\Phi(\mathbf{q})=\frac{1}{2} \boldsymbol{\mu}^{T} \mathbf{W}_{u} \boldsymbol{\mu} & =\left(\mathbf{u}_{2}-\mathbf{R}_{u} \mathbf{q}\right)^{T} \mathbf{W}_{u}\left(\mathbf{u}_{2}-\mathbf{R}_{u} \mathbf{q}\right) \\
& =\underbrace{\mathbf{q}^{T} \mathbf{R}_{u}^{T} \mathbf{W}_{u} \mathbf{R}_{u} \mathbf{q}}_{\text {quadratic }}-\underbrace{2 \mathbf{u}_{2}^{T} \mathbf{W}_{u} \mathbf{R}_{u} \mathbf{q}}_{\text {linear }}+\underbrace{\mathbf{u}_{2}^{T} \mathbf{W}_{u} \mathbf{u}_{2}}_{\text {constant }} .
\end{aligned}
$$

If the quaratic term of this function is convex (i.e. the Hessian matrix $\mathbf{R}_{u}^{T} \mathbf{W}_{u} \mathbf{R}_{u}$ is positive definite), we can simply find the minimum of $\Phi(\mathbf{q})$ by setting its first derivative to zero:

$$
\left(\frac{\partial \Phi}{\partial \mathbf{q}}\right)^{T}=\mathbf{R}_{u}^{T} \mathbf{W}_{u} \mathbf{R}_{u} \mathbf{q}-\mathbf{R}_{u}^{T} \mathbf{W}_{u} \mathbf{u}_{2} \stackrel{!}{=} \mathbf{0},
$$

where we already assumed that $\mathbf{W}_{u}$ is symmetric. This means for the sought transformation matrix $\mathbf{T}_{u}$ :

$$
\mathbf{q}=\underbrace{\left(\mathbf{R}_{u}^{T} \mathbf{W}_{u} \mathbf{R}_{u}\right)^{-1} \mathbf{R}_{u}^{T} \mathbf{W}_{u}}_{\mathbf{T}_{u}} \mathbf{u}_{2}
$$

Where we have now found the 'optimal' solution $\mathbf{q}$ (according to the cost function (11)) to approximate a given $\mathbf{u}_{2}$ with the IDMs. This matrix is the same as the Moore Penrose pseudoinverse in [16].

Remark 2: The positive definiteness of the Hessian matrix shall get some brief attention here. In simple least squares the Hessian matrix is $\mathbf{R}_{u}^{T} \mathbf{R}_{u}$, which is always positive definite if the IDMs contained in $\mathbf{R}_{u}$ are linearly independent (i.e. we chose only such modes that can not be represented by a combination of other modes already contained in the reduced basis $\mathbf{R}_{u}$ ). Thus:

$$
\begin{aligned}
& \mathbf{R}_{u} \mathbf{q} \neq \mathbf{0} \\
& \forall \mathbf{q} \neq \mathbf{0} \\
& \mathbf{q}^{T} \mathbf{R}_{u}^{T} \mathbf{R}_{u} \mathbf{q}=: \mathbf{x}^{T} \mathbf{x}>0 ; \\
& \forall \mathbf{q} \neq \mathbf{0}
\end{aligned}
$$

Now the same trick to show/ensure the positive definiteness of the Hessian matrix, can be applied if we choose the weighting matrix $\mathbf{W}_{u}$ as a symmetric positive definite matrix. Then $\mathbf{W}_{u}$ can be factored in its (all positive!) eigenvalues $\boldsymbol{\Lambda}=\operatorname{diag}\left(\lambda_{i i}\right)$ and the orthonormal eigenvectors $\mathbf{Q}$. Thus:

$$
\begin{aligned}
\mathbf{q}^{T} \mathbf{R}_{u}^{T} \mathbf{W}_{u} \mathbf{R}_{u} \mathbf{q} & =\mathbf{q}^{T} \mathbf{R}_{u}^{T} \mathbf{Q} \boldsymbol{\Lambda} \mathbf{Q}^{T} \mathbf{R}_{u} \mathbf{q} \\
& =\mathbf{q}^{T} \mathbf{R}_{u}^{T} \mathbf{Q} \sqrt{\boldsymbol{\Lambda}} \sqrt{\boldsymbol{\Lambda}} \mathbf{Q}^{T} \mathbf{R}_{u} \mathbf{q} \quad=: \mathbf{x}^{T} \mathbf{x}>0 ; \quad \forall \mathbf{q} \neq \mathbf{0}
\end{aligned}
$$

Obviously we could also add multiple weighting matrices to include different quality criteria in our transformation, e.g. $\mathbf{W}_{u}=\mathbf{W}_{u, 1}+\mathbf{W}_{u, 2}$; as long as $\mathbf{W}_{u, 1}$ and $\mathbf{W}_{u, 2}$ are also symmetric positive definite.

\subsubsection{Displacement weighting matrices}

We will now discuss some possibilities for choosing a weighting matrix $\mathbf{W}_{u}$ in the transformation $\mathbf{T}_{u}$.

\section{Testing experience}

In experimental practice it is often the case that some measurement channels are more prone to errors than others. This may be due to a loose connection with the structure, internal sensor damage or electromagnetic compatibility issues. Another example are sensors that cannot be positioned as accurately as others. The positioning and orientation of the sensors plays an important role in the quality of the transformation (see e.g. ${ }_{I} \mathbf{r}^{k}$ and ${ }_{I} \mathbf{e}_{x}^{k}$ in eq. (8)). An example for this importance can be found in [19, pp.63-65].

For one of these reasons, the experimentalist may feel that this/these specific sensor channels (let us call them $s$ ) may not be trusted as much as the other channels. Hence, minimizing their error $\mu_{s}$ must not be emphasized as much in the transformation. The weighting matrix $\mathbf{W}_{u}$ can thus be chosen as a diagonal matrix with lower weights for the untrusted sensors, e.g.:

\footnotetext{
${ }^{2}$ Also frequently called weighted least squares [18].
} 


$$
\begin{array}{r}
\mathbf{W}_{u}=\operatorname{diag}\left(W_{i i}\right) \quad \text { with } \quad W_{i i}=1, \quad \text { for } \quad i \neq s \\
0<W_{i i}<1, \quad \text { for } i=s
\end{array}
$$

\section{Statistics}

In statistics it is common to automatically give low weights to uncertain sensor channels, by using their inverse covariance matrix as weighting: $\mathbf{W}_{u}=\boldsymbol{\Sigma}_{u_{2}}^{-1}$. It can be shown [18, pp.200-216] that, when choosing the inverse covariance matrix of the sensor channels as $\mathbf{W}_{u}$ in the transformation to $\mathbf{q}$, one is effectively minimizing all entries in the covariance matrix of the outputs $\boldsymbol{\Sigma}_{q}$.

The basic idea of this approach becomes obvious if we assume that the errors in $\mathbf{u}_{2}$ are uncorrelated, i.e. $\boldsymbol{\Sigma}_{u_{2}}=\operatorname{diag}\left(\sigma_{i i}^{2}\right)$ is a diagonal matrix containing the squared variances in the single sensor channels. Using $\mathbf{W}_{u}=\boldsymbol{\Sigma}_{u_{2}}^{-1}$ is then simply putting less weight on those sensor channels with a high variance $\sigma_{i i}$.

Notice, that when transforming an FRF matrix $\mathbf{Y}_{q 2}=\mathbf{T}_{u} \mathbf{Y}_{22}$, one is using the same transformation for each column of the matrix. We will denote the column $j$ by $\mathbf{Y}_{22}(:, j)$. Each column $j$ respresents the response in the sensor channels $\mathbf{u}_{2}$ to a unit force input in $j$ and will have a specific error variance. Thus, we might want to transform each column $j$ separately, using a different weighting matrix which is representing the sensor errors for this force input.

We will now try to come up with a transformation specifically suited to experimental dynamics, by using the same ideas as in statistics. A common quantity to indicate the reliability of a measured FRF matrix entry $Y(i, j)$ is its coherence $\gamma(i, j)$, where $0 \leq \gamma(i, j) \leq 1$. The coherence is typically low at frequencies where a force input in $j$ is exciting only a small motion in sensor channel $i$, so that a large part of the measured sensor signal is due to background noise and may thus not be trusted as much. The coherence is also a standard output, being calculated by most experimental modal analysis softwares, which may facilitate implementation of a statistical criterion that is based on the coherence. A straightforward approach to transform the FRF matrix, would be to utilize the (e.g. squared) coherences of a FRF matrix column $\mathbf{Y}_{22}(:, j)$ as a diagonal weighting matrix for the transformation of this matrix column:

$$
\begin{aligned}
& \mathbf{Y}_{q 2}(:, j)=\underbrace{\left(\mathbf{R}_{u}^{T} \mathbf{W}_{u, j} \mathbf{R}_{u}\right)^{-1} \mathbf{R}_{u}^{T} \mathbf{W}_{u, j}}_{\mathbf{T}_{u, j}} \mathbf{Y}_{22}(:, j) \quad \text { with, } \\
& \mathbf{Y}_{22}(:, j)=\left[\begin{array}{c}
Y_{22}(1, j) \\
Y_{22}(2, j) \\
\vdots
\end{array}\right], \quad \mathbf{W}_{u, j}=\left[\begin{array}{lll}
\gamma^{2}(1, j) & & \\
& \gamma^{2}(2, j) & \\
& & \ddots
\end{array}\right]
\end{aligned}
$$

Notice that the weighting matrix in this case, is changing for each measured frequency $\omega$, since also the coherence is changing for each measured frequency. It is thereby changing the 'trusted' sensor channels depending on the frequency, since at some frequencies a specific sensor channel might be in an antiresonance with very low signal to noise ratio but be valuable in other frequency regions. This might be an advantage over using a fixed weighting matrix as in eq. (14).

\section{Energy minimization}

When considering the transformation error in the displacements $\boldsymbol{\mu}$, it is a natural choice to minimize the energy produced by this error, i.e.:

$$
\text { Minimize: } \Phi(\mathbf{q})=\boldsymbol{\mu}^{T} \mathbf{Z}_{22} \boldsymbol{\mu}
$$

where $\mathbf{Z}_{22}$ denotes the impedance matrix in the interface dofs $\mathbf{u}_{2}$. As will be seen in section 2.3 , the coupling of two transformed FRF matrices effectively means a weakening of the interface compatibility condition, i.e. the residuals of the transformation $\boldsymbol{\mu}$ on either side of the interface are left uncoupled. A minimization as shown above would therefore ensure minimal energy transmission loss over the interface.

However there are also some practical problems associated to choosing $\mathbf{W}_{u}=\mathbf{Z}_{22}$. The first issue is the availability of $\mathbf{Z}_{22}$, since it would require measuring a driving point admittance in all sensor dofs on the interface, i.e. excitation forces in positions and directions equal to the sensor dofs. This may not be possible in all cases (exciting on the sensor faces directly is not recommended, see e.g. [15]). This may be overcome at the expense of building a finite element model of the interface, and thereby obtaining a symmetric $\mathbf{Z}_{22}$ in the interface dofs.

Another problem is more related to the noise pollution of the sensor signals. Typically the displacement response in those directions that are very stiff (i.e. producing a high energy) is also low. Thus, one would effectively over-emphazise those components of $\mathbf{u}_{2}$ in the in the transformation, that already have a lower signal to noise ratio. 


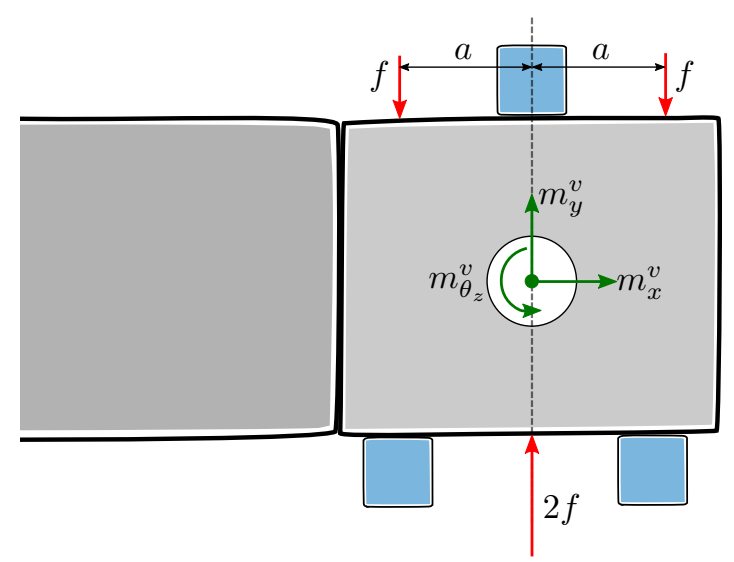

Figure 4: Exemplary nullspace vector for input forces.

\subsubsection{Force modes}

In essence we have to make the same kinematic assumptions for representing the forces as for the displacements. The number of force inputs $n_{f}$ is typically higher than the number of IDMs $p$. Their position must also be carefully chosen, so that all IDMs are excited [16].

$$
\mathbf{f}_{\mathbf{2}} \in \mathbb{C}^{n_{f}}, \quad \mathbf{m} \in \mathbb{C}^{p}, \quad \text { with } \quad p \leq n_{f} .
$$

Let us consider again the example in figure 3, with a locally rigid interface on virtual point $v$. All input forces $\mathbf{f}_{2}$ will result in a generalized force $\mathbf{m}_{t}^{v}$ and a generalized moment $\mathbf{m}_{\theta}^{v}$. For one single force input $f^{h}$ this can be expressed by:

$$
\mathbf{m}^{v}=\left[\begin{array}{l}
\mathbf{m}_{t}^{v} \\
\mathbf{m}_{\theta}^{v}
\end{array}\right]=\left[\begin{array}{c}
{ }_{I} \mathbf{e}^{h} \\
{ }_{I} \mathbf{r}^{k} \times{ }_{I} \mathbf{e}^{h}
\end{array}\right] f^{h}=\mathbf{R}_{f}^{T} f^{h},
$$

where $\mathbf{R}_{f^{h}}^{T}$ denotes the $6 \times 1$ matrix representing the virtual point load $\mathbf{m}^{v}$ resulting from a unit force input in $f^{h}$ and ${ }_{I} \mathbf{e}^{h}$ denotes the direction vector in which the force was applied. The virtual point loads resulting from the other force inputs can be found following a similar procedure. Putting each contribution in a column of $\mathbf{R}_{f}^{T}$, we can write

$$
\mathbf{m}=\mathbf{R}_{f}^{T} \mathbf{f}_{2} \quad \text { with } \quad \mathbf{R}_{f}^{T} \in \mathbb{R}^{p \times n_{f}} .
$$

Notice that $\mathbf{R}_{f}^{T}$ is written transposed since it would directly correspond to the transpose of $\mathbf{R}_{u}$ in eq. (9) if we were measuring driving point FRFs only, i.e. if all force inputs would have a corresponding sensor channel in the same geometric position and in the same direction. However, it is a great practical advantage that different dofs can be used for force inputs and displacement outputs.

\subsubsection{Force transformation}

In order to transform a measured FRF matrix according to eq. (6) we need to find a force transformation matrix $\mathbf{T}_{f}^{T}$ that transforms a given load in the generalized forces $\mathbf{m}$, to an equivalent vector of input forces $\tilde{\mathbf{f}}_{2}$ :

$$
\begin{array}{ll} 
& \tilde{\mathbf{f}}_{2}=\mathbf{T}_{f}^{T} \mathbf{m}, \\
\text { where: } & \mathbf{R}_{f}^{T} \tilde{\mathbf{f}}_{2} \stackrel{!}{=} \mathbf{m} .
\end{array}
$$

The problem in eq. (20) and (21) is fundamentally different from the displacement transformation, stated in eq. (10). The requirement in eq. (21) is an underdetermined problem and there are infinitely many possible solutions for $\tilde{\mathbf{f}}_{2}$ that fulfill this requirement. Any vector in the nullspace of $\mathbf{R}_{f}^{T}$, lets call an example vector $\tilde{\mathbf{f}}_{2, n u l l}$, could be added to $\tilde{\mathbf{f}}_{2}$ to produce yet another possible solution to eq. (21). For the assumption of a locally rigid interface, all $\tilde{\mathbf{f}}_{2, \text { null }}$ would be exciting only flexible modes. Figure 4 shows a depiction of such a possible $\tilde{\mathbf{f}}_{2, n u l l}$. 
The question for the transformation now remains: how do we find a solution $\tilde{\mathbf{f}}_{2}$ from those possible combinations, which is 'good' from an engineering point of view? We can state a standard convex optimization problem where we seek to minimize a scalar cost function $\Phi\left(\tilde{\mathbf{f}}_{2}\right)$, while the optimum solution $\tilde{\mathbf{f}}_{2}$ is subject to constraints $\mathbf{g}\left(\tilde{\mathbf{f}}_{2}\right)$ :

$$
\begin{array}{cl}
\text { Minimize: } & \Phi\left(\tilde{\mathbf{f}}_{2}\right)=\frac{1}{2} \tilde{\mathbf{f}}_{2}^{T} \mathbf{W}_{f} \tilde{\mathbf{f}}_{2}, \\
\text { Subject to: } & \mathbf{g}\left(\tilde{\mathbf{f}}_{2}\right)=\mathbf{R}_{f}^{T} \tilde{\mathbf{f}}_{2}-\mathbf{m}=\mathbf{0},
\end{array}
$$

where we made use of the force weighting matrix $\mathbf{W}_{f}$. Now following the same arguments as in the transformation of the displacements, we can state that $\Phi\left(\tilde{\mathbf{f}}_{2}\right)$ has only one global minimum, if the weighting matrix $\mathbf{W}_{f}$ is positive definite. We then put this together in the standard optimization formulation for finding a constrained minimum, i.e. forming the Lagrangian $\mathcal{L}\left(\tilde{\mathbf{f}}_{2}, \boldsymbol{\lambda}\right)$ and search for its stationary point:

$$
\begin{aligned}
\mathcal{L}\left(\tilde{\mathbf{f}}_{2}, \boldsymbol{\lambda}\right) & =\Phi\left(\tilde{\mathbf{f}}_{2}\right)+\boldsymbol{\lambda}^{T} \mathbf{g}\left(\tilde{\mathbf{f}}_{2}\right) \\
\left(\frac{\partial \mathcal{L}}{\partial \tilde{\mathbf{f}}_{2}}\right)^{T} & =\mathbf{W}_{f} \tilde{\mathbf{f}}_{2}+\mathbf{R}_{f} \boldsymbol{\lambda} \stackrel{!}{=} \mathbf{0} \\
\left(\frac{\partial \mathcal{L}}{\partial \boldsymbol{\lambda}}\right) & =\mathbf{R}_{f}^{T} \tilde{\mathbf{f}}_{2}-\mathbf{m} \stackrel{!}{=} \mathbf{0}
\end{aligned}
$$

where eq. (26) is basically enforcing the constraints. Equation (25) states that at the optimum the gradient of $\Phi\left(\tilde{\mathbf{f}}_{2}\right)$ can be expressed as a linear combination (determined by $\boldsymbol{\lambda}$ ) of the constraint function gradients. This effectively means that at the optimum $\tilde{\mathbf{f}}_{2}$ there is no 'direction' $\Delta \tilde{\mathbf{f}}_{2}$ that could improve the value of $\Phi\left(\tilde{\mathbf{f}}_{2}\right)$ without changing/violating one of the constraints in $\mathbf{g}\left(\tilde{\mathbf{f}}_{2}\right)=\mathbf{0}$ (see e.g. [18, pp.603ff] or any standard text on optimization).

We can then use equation (25) to solve for $\tilde{\mathbf{f}}_{\mathbf{2}}=-\mathbf{W}_{f}^{-1} \mathbf{R}_{f} \boldsymbol{\lambda}$ and insert this in equation (26) to solve for the Lagrange multipliers $\lambda$, yielding:

$$
\lambda=-\left(\mathbf{R}_{f}^{T} \mathbf{W}_{f}^{-1} \mathbf{R}_{f}\right)^{-1} \mathbf{m}
$$

which, reinserted in eq. (25), means for the 'optimal' solution $\tilde{\mathbf{f}}_{2}$ :

$$
\tilde{\mathbf{f}}_{2}=\underbrace{\mathbf{W}_{f}^{-1} \mathbf{R}_{f}\left(\mathbf{R}_{f}^{T} \mathbf{W}_{f}^{-1} \mathbf{R}_{f}\right)^{-1}}_{\mathbf{T}_{f}^{T}} \mathbf{m},
$$

where we call $\mathbf{T}_{f}^{T}$ the transformation matrix for transforming a VP load $\mathbf{m}$ to an equivalent $\tilde{\mathbf{f}}_{2}$ in the force dofs (which we actually physically measured in $\mathbf{Y}_{22}$ ). Note that it is particularly easy to verify that this solution $\tilde{\mathbf{f}}_{2}$ satisfies the constraints eq. (23). The above transformation matrix can also directly be seen as a right MoorePenrose pseudoinverse [16].

\subsubsection{Force weighting matrices}

We will now explain some common choices for the force weighting matrix $\mathbf{W}_{f}$, which is in large parts similar to the discussion for the displacements, but differs in some details.

\section{Testing experience}

In practice some impacts in the set of measurements might be trusted less than the other impacts, let us denote them with $s$. One reason could be that the space for applying a hammer impact was very narrow and putting in enough excitation energy was difficult or most often yielded a double impulse. For badly accessible impact positions, it is also hard to assure that the point is hit exactly in the assumed position ${ }_{I} \mathbf{r}^{j}$ and $\operatorname{direction}{ }_{I} \mathbf{e}^{j}$ (see figure 3). This may result in a bad coherence for the measured column $s$ in the FRF matrix, $\mathbf{Y}_{22}(:, s)$. Overall, many engineering reasons might yield the conclusion that the solution $\tilde{\mathbf{f}}_{2}$ in eq. (21) should better contain less contribution of scalar forces in $s$ and make more use of the other 'trusted' force dofs. This criterion might be included by using the following weighting matrix:

$$
\begin{array}{lll}
\mathbf{W}_{f}=\operatorname{diag}\left(W_{i i}\right) \quad \text { with } & W_{i i}=1 & \text { for } i \neq s, \\
& W_{i i}>1 & \text { for } i=s
\end{array}
$$


This way the particular entries $\tilde{\mathbf{f}}_{2}(s, 1)$ are 'penalized' in the search for the minimum, since they lead to a more rapid growth of the quality measure $\Phi\left(\tilde{\mathbf{f}}_{2}\right)$ when compared to the other force inputs $\tilde{\mathbf{f}}_{2}(i, 1) \quad i \neq s$.

\section{Statistics}

Similar as for the displacements, it might be desired to choose a frequency dependent optimality criterion also for the force transformation. In order to account for the statistical reliability of the FRF matrix entries we can again take the the coherence $\gamma(i, j)$ as an indicator. When performing the transformation to generalized forces, $\mathbf{Y}_{2 m}=\mathbf{Y}_{22} \mathbf{T}_{f}^{T}$, one is effectively transforming each row of the matrix. That means the response of a single sensor channel $i$ to a combination of all measured force inputs $\mathbf{f}_{2}$ (contained in row $\mathbf{Y}_{22}(i,:)$ ) is transformed to the response of channel $i$ to a combination of the virtual point loads $\mathbf{m}$ (contained in row $\mathbf{Y}_{2 m}(i,:)$ ). Since the coherence differs for each channel $i$ it is desirable to transform each row of the FRF matrix separately:

$$
\begin{aligned}
& \mathbf{Y}_{2 q}(i,:)=\mathbf{Y}_{22}(i,:) \underbrace{\mathbf{W}_{f, i}^{-1} \mathbf{R}_{f}\left(\mathbf{R}_{f}^{T} \mathbf{W}_{f, i}^{-1} \mathbf{R}_{f}\right)^{-1}}_{\mathbf{T}_{f, i}^{T}} \text { with, } \\
& \mathbf{Y}_{22}(i,:)=\left[\begin{array}{c}
Y_{22}(i, 1) \\
Y_{22}(i, 2) \\
\vdots
\end{array}\right], \quad \mathbf{W}_{f, i}^{-1}=\left[\begin{array}{lll}
\gamma(i, 1)^{2} & & \\
& \gamma(i, 2)^{2} & \\
& & \ddots
\end{array}\right] .
\end{aligned}
$$

This proposed transformation would automatically prefer the 'trusted'/high coherence force inputs at each frequency over the force inputs with a low coherence. Thus the equivalent force $\tilde{\mathbf{f}}_{2}$ for a given generalized force $\mathbf{m}$ would contain less contribution of the 'untrusted' force inputs.

\section{Energy minimization}

When minimizing a cost function $\Phi\left(\tilde{\mathbf{f}}_{2}\right)$, it is a natural choice to minimize the energy produced by the equivalent input forces $\tilde{\mathbf{f}}_{2}$, i.e.:

$$
\text { Minimize: } \quad \Phi\left(\tilde{\mathbf{f}}_{2}\right)=\tilde{\mathbf{f}}_{2}^{T} \mathbf{Y}_{22} \tilde{\mathbf{f}}_{2},
$$

where $\mathbf{Y}_{22}$ denotes the admittance matrix in the interface force dofs $\tilde{\mathbf{f}}_{2}$.

Notice that the transformation in eq. (28) requires only the inverse of the force weighting matrix. So effectively, the same practical problems associated to choosing $\mathbf{W}_{f}=\mathbf{Y}_{22}=\mathbf{Z}_{22}^{-1}$ as in the energy minimization for the displacements in section 2.2.3 apply here.

\subsection{Weakening of the compatibility condition}

In the last section we have derived the transformation matrices necessary for transforming the interface dofs $\mathbf{u}_{2}, \mathbf{f}_{2}$ on the generalized dofs $\mathbf{q}, \mathbf{m}$. The interface transformation of substructure $A$ 's full admittance matrix $\mathbf{Y}^{A}$ may thus be written as:

$$
\tilde{\mathbf{Y}}^{A}=\left[\begin{array}{cc}
\mathbf{Y}_{11}^{A} & \mathbf{Y}_{1 m}^{A} \\
\mathbf{Y}_{q 1}^{A} & \mathbf{Y}_{q m}^{A}
\end{array}\right]=\left[\begin{array}{cc}
\mathbf{I} & \mathbf{0} \\
\mathbf{0} & \mathbf{T}_{u}^{A}
\end{array}\right]\left[\begin{array}{cc}
\mathbf{Y}_{11}^{A} & \mathbf{Y}_{12}^{A} \\
\mathbf{Y}_{21}^{A} & \mathbf{Y}_{22}^{A}
\end{array}\right]\left[\begin{array}{cc}
\mathbf{I} & \mathbf{0} \\
\mathbf{0} & \left(\mathbf{T}_{f}^{A}\right)^{T}
\end{array}\right]
$$

where $\tilde{\mathbf{Y}}^{A}$ denotes the transformed substructure matrix. An analogous expression would yield $\tilde{\mathbf{Y}}^{B}$, the transformed admittance matrix of substructure $B$. The two transformed matrices can directly be coupled to each other, as shown in section 2.1, since their generalized dofs on either side of the interface are matching:

$$
\tilde{\mathbf{Y}}^{A B}=\tilde{\mathbf{Y}}^{A \mid B}-\tilde{\mathbf{Y}}^{A \mid B} \mathbf{B}^{T}\left(\mathbf{B} \tilde{\mathbf{Y}}^{A \mid B} \mathbf{B}^{T}\right)^{-1} \mathbf{B} \tilde{\mathbf{Y}}^{A \mid B} \quad \text { where } \quad \tilde{\mathbf{Y}}^{A \mid B}=\left[\begin{array}{cc}
\tilde{\mathbf{Y}}^{A} & \mathbf{0} \\
\mathbf{0} & \tilde{\mathbf{Y}}^{B}
\end{array}\right]
$$

We will now show that coupling the transformed matrices of $A$ and $B$ corresponds to a weakening of the interface compatibility condition.

Assume we are coupling the non-transformed (i.e. measured) admittance matrices, written in block diagonal form $\mathbf{Y}^{A \mid B}$ as in eq. (1). However, we are now enforcing the compatibility only in the direction of the generalized dofs $\mathbf{q}$ and allow only reaction forces in the generalized forces $\mathbf{m}$ : 


$$
\begin{aligned}
& \mathbf{Y}^{A \mid B}(\mathbf{f}+\underbrace{\mathbf{B}_{f}^{T} \boldsymbol{\lambda}}_{=\mathbf{B}})=\mathbf{u}, \quad \text { where } \quad \mathbf{B}_{f}=\left[\begin{array}{llll}
\mathbf{0} & \mathbf{T}_{f}^{A} & -\mathbf{T}_{f}^{B} & \mathbf{0}
\end{array}\right], \\
& \mathbf{B}_{u} \mathbf{u}=\mathbf{0}, \quad \text { where } \quad \mathbf{B}_{u}=\left[\begin{array}{llll}
\mathbf{0} & \mathbf{T}_{u}^{A} & -\mathbf{T}_{u}^{B} & \mathbf{0}
\end{array}\right] .
\end{aligned}
$$

Inserting eq. (35) in eq. (36) yields:

$$
\begin{aligned}
\boldsymbol{\lambda} & =-\underbrace{\left(\mathbf{B}_{u} \mathbf{Y}^{A \mid B} \mathbf{B}_{f}^{T}\right)^{-1}}_{Z_{\text {int }}} \underbrace{\mathbf{B}_{u} \mathbf{Y}^{A \mid B} \mathbf{f}}_{\Delta \mathbf{q}} \\
& =-\underbrace{\left(\mathbf{Y}_{q m}^{A}+\mathbf{Y}_{q m}^{B}\right)^{-1}}_{Z_{\text {int }}} \underbrace{\left[\begin{array}{llll}
\mathbf{0} & \mathbf{T}_{u}^{A}-\mathbf{T}_{u}^{B} & \mathbf{0}
\end{array}\right]}_{=\mathbf{B}_{u}} \mathbf{Y}^{A \mid B} \mathbf{f}
\end{aligned}
$$

Reinserting $\boldsymbol{\lambda}$ in eq. (35) yields the 'weakly coupled' admittance $\mathbf{Y}_{\text {weak }}^{A B}$ :

$$
\underbrace{\left(\mathbf{Y}^{A \mid B}-\mathbf{Y}^{A \mid B} \mathbf{B}_{F}^{T}\left(\mathbf{B}_{u} \mathbf{Y}^{A \mid B} \mathbf{B}_{f}^{T}\right)^{-1} \mathbf{B}_{u} \mathbf{Y}^{A \mid B}\right)}_{\mathbf{Y}_{\text {weak }}^{A B}} \mathbf{f}=\mathbf{u} .
$$

Notice that $\mathbf{Y}_{\text {weak }}^{A B}$ has the same size as the non-transformed block matrix $\mathbf{Y}^{A \mid B}$. However, as opposed to $\mathbf{Y}^{A B}$ in eq. (5), when writing down $\mathbf{Y}_{\text {weak }}^{A B}$ in the verbose $4 \times 4$ block matrix notation (indicated in eq. (1)) we would notice that the second and third row are not identical! Meaning that the displacements $\mathbf{u}_{2}^{B}$ and $\mathbf{u}_{2}^{A}$ are not perfectly coupled, but remain uncoupled in the residuals of the transformation $\boldsymbol{\mu}$. Inserting eq. (13) in eq. (10), we can write for the interface residuals $\mu$ :

$$
\boldsymbol{\mu}=\left(\mathbf{I}-\mathbf{R}_{u} \mathbf{T}_{u}\right) \mathbf{u}_{2} .
$$

These 'residual motions' $\boldsymbol{\mu}$ may be present on either side of the interface as a part of the real motion $\mathbf{u}_{2}=\mathbf{R}_{\mathbf{u}} \mathbf{q}+\boldsymbol{\mu}$. However, when transforming the residual parts $\boldsymbol{\mu}$ in eq. (39) on the IDMs we see that of course $\mathbf{T}_{u} \boldsymbol{\mu}=\mathbf{0}$. When looking at the last part of eq. (37) this means that the residual motions on either side are not producing any reaction force $\boldsymbol{\lambda}$ and are thus remaining uncoupled.

This weakly coupled $\mathbf{Y}_{\text {weak }}^{A B}$ may then be transformed to the generalized dofs via:

$$
\tilde{\mathbf{Y}}^{A B}=\left[\begin{array}{cccc}
\mathbf{I} & \mathbf{0} & \mathbf{0} & \mathbf{0} \\
\mathbf{0} & \mathbf{T}_{u}^{A} & \mathbf{0} & \mathbf{0} \\
\mathbf{0} & \mathbf{0} & \mathbf{T}_{u}^{B} & \mathbf{0} \\
\mathbf{0} & \mathbf{0} & \mathbf{0} & \mathbf{I}
\end{array}\right] \mathbf{Y}_{\text {weak }}^{A B}\left[\begin{array}{cccc}
\mathbf{I} & \mathbf{0} & \mathbf{0} & \mathbf{0} \\
\mathbf{0} & \left(\mathbf{T}_{f}^{A}\right)^{T} & \mathbf{0} & \mathbf{0} \\
\mathbf{0} & \mathbf{0} & \left(\mathbf{T}_{f}^{B}\right)^{T} & \mathbf{0} \\
\mathbf{0} & \mathbf{0} & \mathbf{0} & \mathbf{I}
\end{array}\right]
$$

which can be verified to be the same matrix $\tilde{\mathbf{Y}}^{A B}$ that we would have obtained if we had directly coupled the reduced matrices (see eq. (34)). This result is pointing out two important assumptions that the engineer has to make when coupling two admittances that were projected on IDMs (eq. (34)):

- Substructures are left uncoupled in the directions not contained in the IDMs.

- All important motion is contained in the IDMs and thus external excitations and motions in directions not contained in the IDMs are negligible or not of special interest.

The result of eq. (38) could also be used to purposely couple two substructures only in predefined directions or modes while leaving their full set of dofs in the equation.

\section{Conclusion}

In this paper we derived the weak compatibility in the coupling of transformed FRFs from a minimization point of view. The use of weighting matrices in the transformation shall be further explored in the future. We have also shown that the coupling of projected FRFs in fact corresponds to a weakening of the interface compatibility 
condition. The findings of past publications, such as 'that the lack of RDOF data underestimates in frequency the correct predictions [of the coupled system resonances, M.H.].' [9], can be clearly attributed to the weakening effect on the interface compatibility when leaving out the rotational IDMs. However this weakening can also be used to purposely keep certain modes uncoupled (e.g. in structures containing relatively loose joints in certain directions). Mayes and Allen have impressively shown in [2, 14] that solving the compatibility condition in experimental substructuring only in a least squares way (called 'MCFS' in these publications), can dramatrically improve the results. This is also an encouraging result for the coupling of substructures in a 'weak' manner as in the projection developed in $[16,15,8]$ and described in this contribution. One important assumption for the use of transformed matrices is that the major excitations and deformations can be adequately modeled with the IDMs. This assumption is also important for TPA methods in general [17], since there the whole complexity of a source is described via the forces applied on the interface $\mathbf{f}_{2} / \mathbf{m}$.

\section{Acknowledgments}

This research is supported and funded by the BMW Group, whom we greatly acknowledge for their support. We also would like to thank Maarten van der Seijs for providing an illustration.

\section{References}

[1] D. de Klerk, D. J. Rixen, and S. N. Voormeeren. "General Framework for Dynamic Substructuring: History, Review and Classification of Techniques". In: AIAA Journal 46.5 (2008), pp. 1169-1181. ISSN: 0001-1452. DOI: $10.2514 / 1.33274$.

[2] M. S. Allen and R. L. Mayes. "Comparison of FRF and modal methods for combining experimental and analytical substructures". In: 25th International Modal Analysis Conference (IMAC XXV). 2007.

[3] S. N. Voormeeren and D. J. Rixen. "A family of substructure decoupling techniques based on a dual assembly approach". In: Mechanical Systems and Signal Processing 27.1 (2012), pp. 379-396. ISSN: 08883270. DOI: 10.1016/j.ymssp.2011.07.028.

[4] B. Jetmundsen, R. L. Bielawa, and W. G. Flannelly. "Generalized Frequency Domain Substructure Synthesis". In: Journal of the American Helicopter Society 33.1 (Oct. 1988), pp. 55-64.

[5] J. R. Crowley and A. Klosterman. "Direct structural modification using frequency response functions". In: Proceedings of IMAC II. 1984.

[6] D. J. Ewins. Modal testing: theory and practice. Vol. 15. Research studies press Letchworth, 1984.

[7] K. G. McConnell and P. S. Varoto. Vibration testing: theory and practice. 2nd Editio. John Wiley \& Sons, 2008.

[8] D. de Klerk et al. "Solving the RDoF Problem in Experimental Dynamic Substructuring". In: Proceedings of the 26th International Modal Analysis Conference - IMAC. 2008. ISBN: 9781605600666.

[9] M. L. M. Duarte and D. J. Ewins. "Some Insights into the Importance of Rotational Degrees-of-freedom and Residual Terms in Coupled Structure Analysis". In: Proceedings of the 13th International Modal Analysis Conference. Society for Experimental Mechanics, 1995, pp. 164-170.

[10] R. R. Craig Jr. and A. J. Kurdila. Fundamentals of Structural Dynamics. John Wiley \& Sons, 2006. ISBN: 978-0-471-43044-5.

[11] J. O'Callahan, P. Avitabile, and R. Riemer. "System equivalent reduction expansion process (SEREP)". In: Proceedings of the 7th international modal analysis conference. Vol. 1. Union College Schnectady, NY. 1989, pp. 29-37.

[12] A. Williams, C. Chipman, and P. Avitabile. "Modal and frequency based substructuring using rotational DOF considerations". In: Proceedings of the 26th International Modal Analysis Conference, Orlando, Florida. 2008.

[13] D. Rixen. "How measurement inaccuracies induce spurious peaks in frequency based substructuring". In: Proceedings of the Twenty Sixth International Modal Analysis Conference, Orlando, FL. Society for Experimental Mechanics, Bethel, CT (2008). ISSN: 21915644. URL: http://sem-proceedings.com/26i/sem.orgIMAC - XXVI - Conf - s28p02 - How - Measurement - Inaccuracies - Induce - Spurious - Peaks - Frequency Based.pdf.

[14] R. L. Mayes and E. C. Stasiunas. "Lightly damped experimental substructures for combining with analytical substructures". In: 25th international modal analysis conference (IMAC XXV), Orlando, FL. 2007. 
[15] M. van der Seijs et al. "Validation of Current State Frequency Based Substructuring Technology for the Characterisation of Steering Gear-Vehicle Interaction". In: ed. by R. Mayes, D. Rixen, and M. Allen. Topics in Experimental Dynamic Substructuring, Volume 2: Proceedings of the 31st IMAC, A Conference on Structural Dynamics, 2013. New York, NY: Springer New York, 2014. Chap. 20, pp. 253-266. ISBN: 978-1-4614-6539-3. DOI: 10.1007/978-1-4614-6540-9. URL: http://link. springer. com/10.1007/9781-4614-6540-9.

[16] M. V. van der Seijs et al. "An improved methodology for the virtual point transformation of measured frequency response functions in dynamic substructuring". In: 4th ECCOMAS Thematic Conference on Computational Methods in Structural Dynamics and Earthquake Engineering 2013.June (2013). DOI: 10. 13140/RG.2.1.2715.3126.

[17] M. V. Van Der Seijs, D. De Klerk, and D. J. Rixen. "General framework for transfer path analysis: History, theory and classification of techniques". In: Mechanical Systems and Signal Processing 68-69 (Feb. 2016), pp. 217-244. ISSN: 10961216. DOI: 10.1016/j.ymssp.2015.08.004. URL: http://dx.doi.org/10.1016/ j.ymssp. 2015.08.004\%20http://linkinghub.elsevier.com/retrieve/pii/S0888327015003647.

[18] G. Strang. Computational Science and Engineering. First Edit. Wellesley-Cambridge Press, 2007. ISBN: 9780961408817.

[19] M. V. van der Seijs. "Experimental Dynamic Substructuring, Analysis and Design Strategies for Vehicle Development". PhD Thesis. Delft University of Technology, 2016. 